\title{
Optical harness and receiver for future L-band radiometer
}

Teresa Mengual, P. Villalba, M. Piqueras, E. Rico, J. Ramírez, et al.

Teresa Mengual, P. Villalba, M. A. Piqueras, E. Rico, J. I. Ramírez, R. Vilaseca, A. Catalán, "Optical harness and receiver for future L-band radiometer," Proc. SPIE 11852, International Conference on Space Optics ICSO 2020, 118522T (11 June 2021); doi: 10.1117/12.2599450

SPIE Event: International Conference on Space Optics - ICSO 2021, 2021, Online Only 


\section{International Conference on Space Optics-ICSO 2020}

Virtual Conference

30 March-2 April 2021

Edited by Bruno Cugny, Zoran Sodnik, and Nikos Karafolas
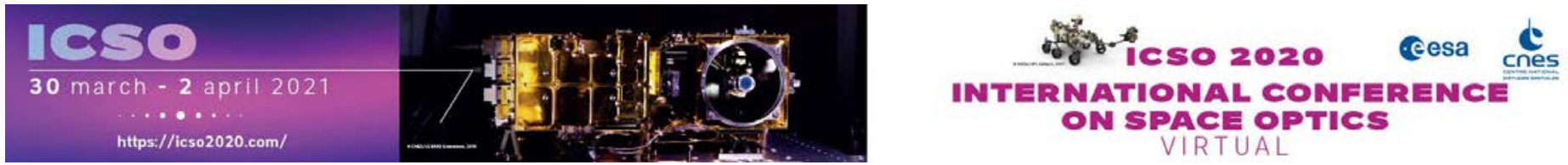

\section{Optical harness and receiver for future L-band radiometer}

\section{Cesa isso procedings denes}




\title{
Optical Harness and Receiver for Future L-Band Radiometer
}

\author{
T. Mengual*a , P. Villalba ${ }^{\mathrm{a}}$, M. A. Piqueras ${ }^{\mathrm{a}}$, E. Rico ${ }^{\mathrm{a}}$, J.I. Ramírez ${ }^{\mathrm{b}}$, R. Vilaseca ${ }^{\mathrm{c}}$, A. Catalán $^{\mathrm{c}}$, \\ ${ }^{a}$ DAS Photonics, Camino de Vera, s/n, Edificio 8F, 46022 Valencia, Spain \\ ${ }^{c}$ Airbus Defence and Space, S.A.U., Avda. Aragón, 404, 28022 Madrid, Spain \\ ${ }^{c}$ SENER Aeroespacial, Parc de l'Alba C/ Creu Casas i Sicart, 86-87, 08290 Cerdanyola, Barcelona, \\ Spain
}

\begin{abstract}
ESA's Soil Moisture and Ocean Salinity (SMOS) mission was launched 2 Nov 2009 being the first ESA satellite relying on a complete optical harness (OHA), which was initially selected for the mechanical properties of optical fiber, what facilitated the deployment of the 3 arms of the instrument. In addition, other interesting advantages of the optical harness played an important role in the instrument performance such as lower propagation losses, immunity to electromagnetic interference, high bandwidth, SWaP reduction, etc. The two main functions of optical harness in SMOS were the distribution of reference clock and the transmission of the IQ data signals.

Based on the good results obtained by SMOS-OPS mission, under the ESA ITI contracts No 4000120740/17/NL/AI and 4000122980/18/NL/IA, DAS Photonics, along with Airbus DS, and SENER Aeroespacial have developed the required OHA and the advanced L-band receivers (ALR), respectively, for a future advanced L-band radiometer mission with a target requirement of $10 \mathrm{~km}$ spatial resolution.

In particular, two OHA configurations have been manufactured and tested. The first configuration aims at solving some identified issues as well as at improving performance of SMOS thanks to lessons learnt from the in-orbit operation, but without attempting novel techniques of calibration or signal distribution. The main goals of the second configuration, based on WDM techniques, are the improvement of the electrical performance and the optimization of the optical harness in terms of layout, i.e, to reduce number of cables/fibres, size, weight, as well as power consumption.
\end{abstract}

Keywords: Interferometer, radiometer, SMOS, optical communications, optical harness

\section{INTRODUCTION}

Soil Moisure and Ocean Salinity (SMOS) belongs to a family of satellite missions that address key scientific challenges identified by the science community and demonstrate breakthrough technology in observing techniques.

The SMOS satellite was launched on November 2, 2009, and carries a novel Microwave Imaging Radiometer with Aperture Synthesis (MIRAS) that operates in the L-band microwave range to capture 'brightness temperature' images.

Interferometry requires that all receivers operate coherently, and thus, if a down-conversion is used, the local oscillator has to be distributed to all antenna elements. Also, if the signals are digitized in the antenna elements, the same sampling clock has to be distributed to all of them, and the output digital streams transferred to the cross correlator need to be synchronous to the receive clock of the correlator. Finally, a common calibration signal is needed at the input of all antenna elements to characterize instrumental errors.

SMOS was the first ESA satellite relying on a complete optical harness, which was initially selected for the mechanical properties of optical fibre, what facilitated the deployment of the three arms of the instrument. In addition, other interesting advantages of the optical harness played an important role in the instrument performance:

- Lower optical fibre transmission losses

*tmengual@dasphotonics.com; phone+34 963556 150; fax +34 963562 581; http://www.dasphotonics.com/ 
- Better phase stability over temperature and bending in comparison with coaxial cable

- Very low electro-magnetic emission levels

- Galvanic isolation between units, as well as insensitivity to ground differential voltages

- Mass and size saving (depending on the case)

Therefore, optical signals transmission and distribution are playing an important role in the ESA future missions. Both digital and analog fiber-optic harnesses have been identified as a key enabling technology to overcome relevant limitations of traditional harness.

Based on the advantages of optical cables and DAS Photonics, along with Airbus DS, and SENER Aeroespacial have developed the required OHA and the advanced L-band receivers (ALR), respectively, as an evolution towards a full optical harness system for a future SMOS Operational (SMOS-OPS) L-band radiometer ${ }^{1,2}$.

\section{PRINCIPLE OF OPERATION AND ARCHITECTURE OF SMOS-OPS}

SMOS objective was to provide global maps of soil moisture and ocean salinity with high accuracy, spatial and temporal resolution which is of great interest in scientific fields as agronomy, climatology, glaciological and meteorological sciences, etc.

SMOS is based on interferometry which allows to obtain an image with a brightness temperature distribution from the cross correlation of pairs of signals captured from multiple antennas looking at the target scene. Therefore the main components needed on board to perform interferometry are (a) a number of antenna elements, (b) a cross correlator and (c) a signal harness in between them.

Interferometry further requires that all receivers operate coherently, and thus, if a down conversion is used, the local oscillator (LO) has to be distributed to all antenna elements. Also, if the signals are digitized in the antenna elements, the same sampling clock has to be distributed to all of them, and the output digital streams transferred to the cross correlator need to be synchronous to the receive clock of the correlator. Finally, a common calibration signal (CAS) is needed at the input of all antenna elements to characterize instrumental errors.

The two main functions of optical harness in SMOS were the distribution of reference clock and the transmission of the IQ data signals. However, a good improvement would be to reduce the extensive use of coaxial cable required to distribute LO and CAS signals, as well as to distribute the signal from a central LO to all the receivers in order to avoid frequent LO phase calibration ${ }^{3,4}$.

The summary of the signals to be distributed in SMOS-OPS Optical Harness (OHA), according to the requirements shown in table 1, are the following:

- Local Oscillator (LO) in L-band at $1384.65 \mathrm{MHz}$ with the possibility to be a sinusoidal signal or a digital square with $50 \%$ duty cycle. This LO signal is transmitted from LO unit to the $\mathrm{N}$ antenna elements.

- Centralized calibration noise signal, an uplink of a $27 \mathrm{MHz}$ L-band calibration noise centered at $1413.5 \mathrm{MHz}$. This signal is transmitted from the Calibration Unit to the $\mathrm{N}$ antenna elements.

- Centralized uplink of the sampling clock, a $115.3875 \mathrm{MHz}$ signal (digital over-sampling clock signal). This sampling clock is transmitted from the Sampling Clock Unit to the $\mathrm{N}$ antenna elements. Alternatively, the sampling clock could be generated locally at the antenna elements by a 12:1 frequency division of the uplinked LO digital signal.

- Downlink output data stream from all antenna elements. The data stream consists in a multiplexed 1-bit sample stream at a rate of $230.775 \mathrm{Mbps}$. 
Table 1. OHA technical specifications for a future advanced L-band radiometer mission

\begin{tabular}{|c|c|}
\hline & Specification \\
\hline \multirow{2}{*}{ Power level spec LO signal } & $-35 \mathrm{dBm}(\mathrm{min})$ \\
\hline & $-25 \mathrm{dBm}(\max )$ \\
\hline LO signal output level accuracy & $<2.2 \mathrm{~dB}$ \\
\hline \multirow{3}{*}{ LO signal Phase Noise } & $<-100 \mathrm{dBc} / \mathrm{Hz}(100 \mathrm{KHz})$ \\
\hline & $<-110 \mathrm{dBc} / \mathrm{Hz}(1 \mathrm{MHz})$ \\
\hline & $\begin{array}{l}<-105 \mathrm{dBc} / \mathrm{Hz} \quad(1400- \\
1427 \mathrm{MHz})\end{array}$ \\
\hline LO Group delay & $< \pm 47 \mathrm{ps}$ \\
\hline \multirow{2}{*}{ Power level spec CAS signal } & $-98.6 \mathrm{dBm}$ (warm) \\
\hline & $-91.8 \mathrm{dBm}($ hot $)$ \\
\hline $\begin{array}{c}\text { CAS signal output level } \\
\text { imbalance }\end{array}$ & $<2.2 \mathrm{~dB}$ \\
\hline CAS Phase & $\leq 3^{\circ}$ \\
\hline $\begin{array}{l}\text { CLK Delay difference between } \\
\text { links }\end{array}$ & $< \pm 500 \mathrm{ps}$ \\
\hline CLK signal Jitter & $< \pm 75 \mathrm{ps}$ \\
\hline CLK Duty cycle (\%) & $40 / 60$ to $60 / 40$ \\
\hline $\begin{array}{c}\text { Data links Bit Error Rate } \\
\text { (BER) }\end{array}$ & $<1 \mathrm{E}-10$ \\
\hline
\end{tabular}

Therefore, the preliminary SMOS-OPS Payload architecture is composed by the following parts (see Figure 1):

- The L-Band Radiometer consisting of $\mathrm{N}$ receivers (antenna elements) which detect the natural emission of earth in L-Band

- The Correlator which cross correlates the received signals from all pairs of Radiometer antenna elements

- The LO signal generation Unit

- The Calibration signal generation Unit

- The Sampling Clock signal generation Unit

- The Correlator, LO, Calibration and Sampling Clock Units can be physically located at the Correlator and Control Unit (CCU)

- The Optical Harness conveying in total 4 signals between the N Radiometer antenna elements and the Correlator / LO /Calibration / Sampling Clock, which will be divided in three main parts (see Figure 1):

- Optical Transceivers in the Correlator Module: the part interfacing with the Calibration, Sampling Clock, Local Oscillator and Correlator Units; this part includes the electrical to optical conversion of the LO signal, the Calibration Signal and the Sampling Clock signal; this part includes also the O/E conversion of the data stream from the antenna elements. 
- Optical Transceivers in the Antenna Element Modules: the part interfacing with the Antenna Elements; this part includes the O/E conversion of the LO signal, the Calibration Signal and the Sampling Clock signal (if required); this part also includes the $\mathrm{E} / \mathrm{O}$ conversion of the data stream output of the antenna elements as well as the derivation of the Sampling Clock signal in the antenna element from the LO signal (if required).

- The Optical Network: the harness connecting the two modules above, implementing the uplink and downlink of the optical signals as required.

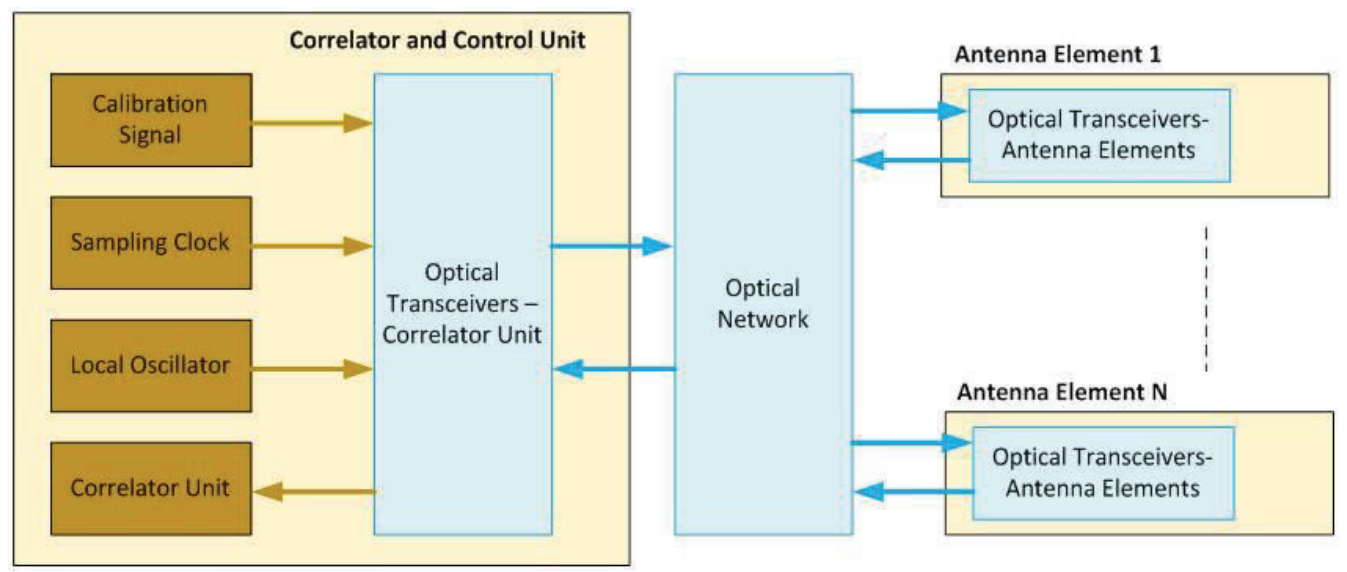

Figure 1. Scheme of the OHA

Different radiometer layout configurations have been considered during the study of the SMOS Operational mission ${ }^{4}$. As a result, two main possible configurations of the SMOS-OPS Radiometer are envisaged.

- Y-shape (i.e. 3 branches) with aprox $1 \mathrm{~m}$ hub and $5.4 \mathrm{~m}$ arms, the hub having 18 antenna elements and each arm consisting of 3 segments of $1.8 \mathrm{~m}$ with 12 antenna elements/segment, i.e. $18+12 \times 3 \times 3=126$ antenna elements in total. This is a larger version of the current SMOS design (see Figure 2)

- Hexagon (i.e. 6 branches) with $6.5 \mathrm{~m}$ sides, each side consisting of 2 segments of 3.25 m with 22 antenna elements/segment, i.e. $22 \times 2 \times 6=264$ antenna elements in total (see Figure 2)

The study of the different OHA configurations has been performed considering the Hexagon-shape and worst case:

- 12 segments of 3.25 meters

- 22 antennas per segment

- 264 antenna elements

- Maximum distance of optical fibre between segments: 20 meters

- Maximum distance in each segment: 2 meters

- Splitting ratio from the hub to the segments: 12

- Splitting ratio in each segment: 22. 

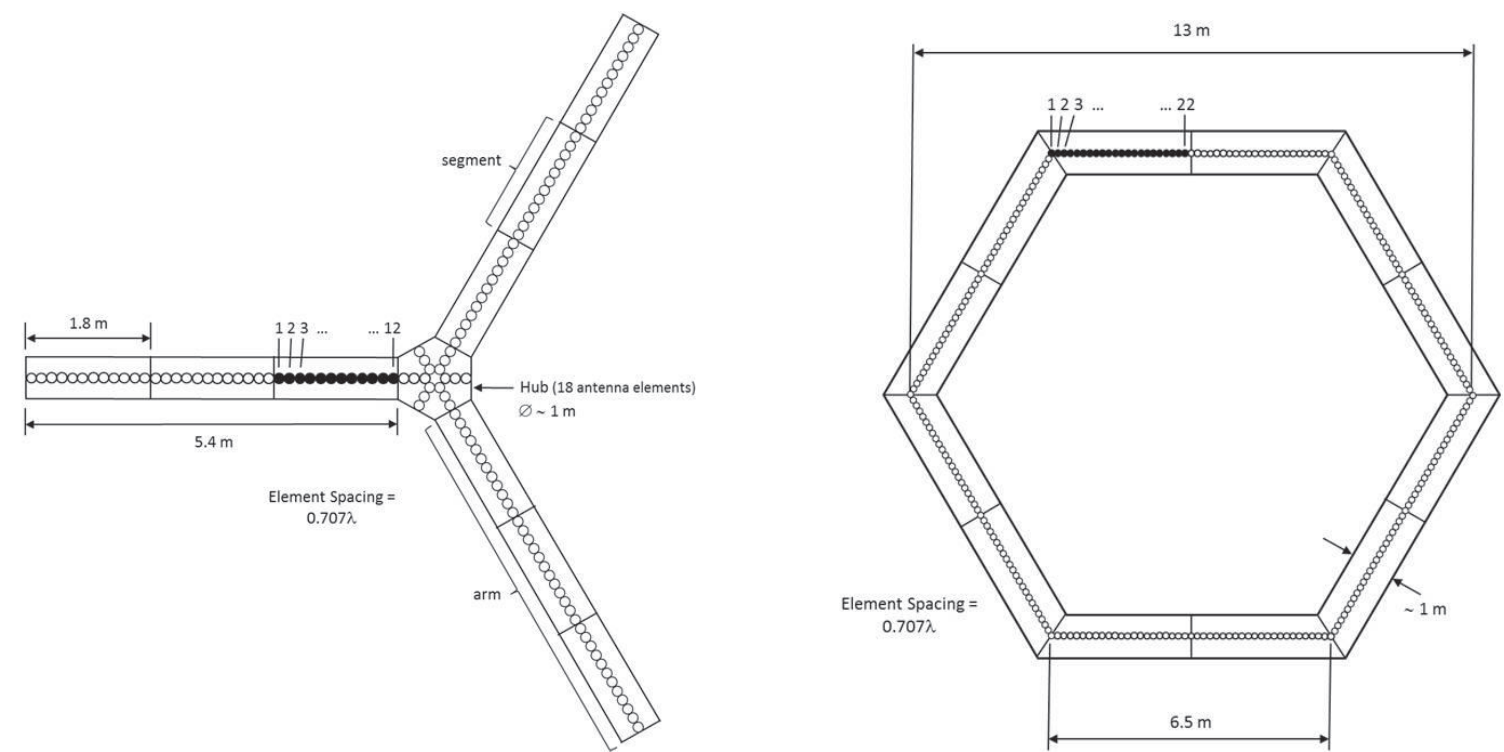

Figure 2. Y-shape and hexagonal version of an SMOS-OPS radiometer instrument

\section{OPTICAL HARNESS BREADBOARD}

A trade-off over 20 different Optical Harness designs has been carried out to select the two most promising options ${ }^{5}$ to design, manufacture and test a breadboard of representative parts of an advanced optical communication system for future SMOS-OPS L-band interferometric radiometer. In particular, two OHA configurations have been manufactured and tested.

The first configuration aims at solving some identified issues as well as at improving performance of SMOS thanks to lessons learnt from the in-orbit operation, but without attempting novel techniques of calibration or signal distribution. The main goals of the second configuration, based on alternative techniques such us wavelength division multiplexing (WDM), are the improvement of the electrical performance and the optimization of the optical harness in terms of layout, i.e, to reduce number of cables/fibres, size, weight, as well as power consumption.

Figure 3 shows a schematic diagram of the proposed OHA for configuration 1. The main advantages of this configuration are the reduction of coaxial cable required, since the LO and CAS signals are distributed in the optical domain, and the distribution of the signal from a central LO to all the receivers to avoid frequent LO phase calibration. As can been seen, this configuration requires one fibre per signal and segment where the CAS and LO have been considered as analog signals and the DATA and CLK as digital signals.

Figure 4 shows a schematic diagram of the proposed OHA for configuration 2. Since this configuration is based on wavelength division multiplexing in the optical domain, the CAS, LO and CLK signals are transmitted as analog signals over a single optical fibre (one per segment) where the CAS and LO have been considered as analog signals and CLK as digital signals. The data signal is distributed in the same way as in configuration 1 . In contrast with configuration 1 , the splitting at segment level is carried out in the optical domain so an optical amplifier is required to maximize the optical power.

Regarding redundancy, SMOS mission has been taken as a reference where redundancy was implemented in the units that affect more than one channel. Passive optical splitters were an exception to this rule due its high reliability. 


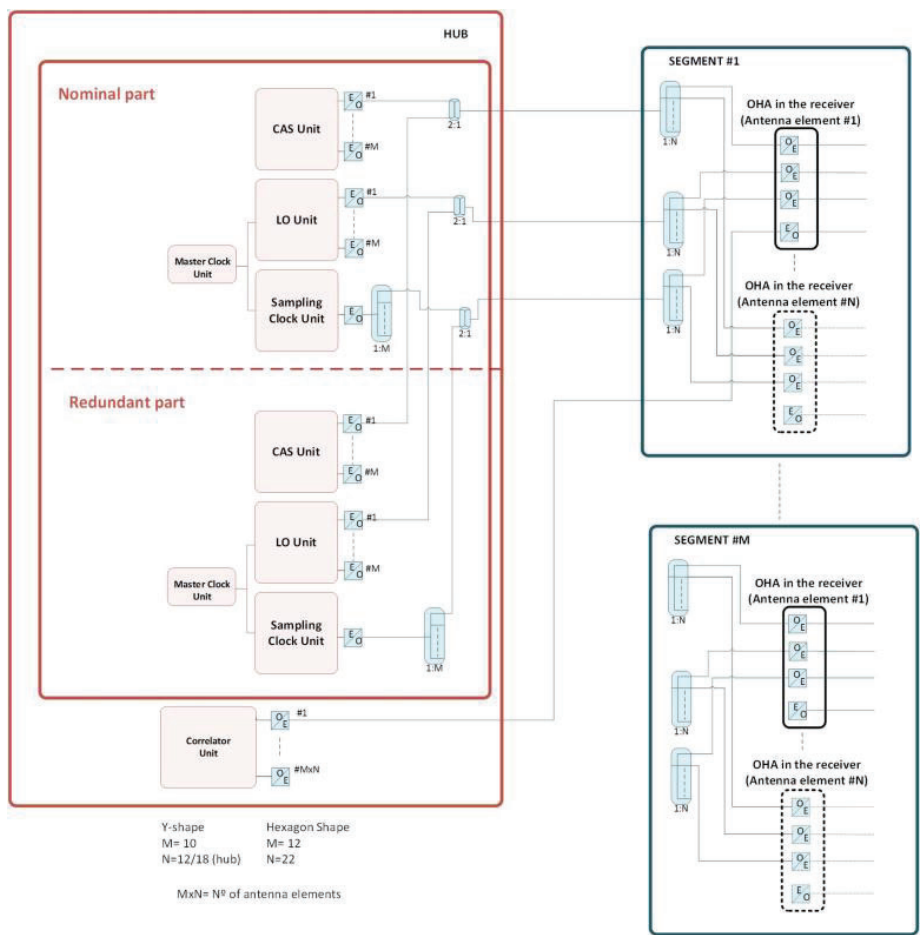

Figure 3. Configuration 1: Schematic diagram of the proposed OHA.

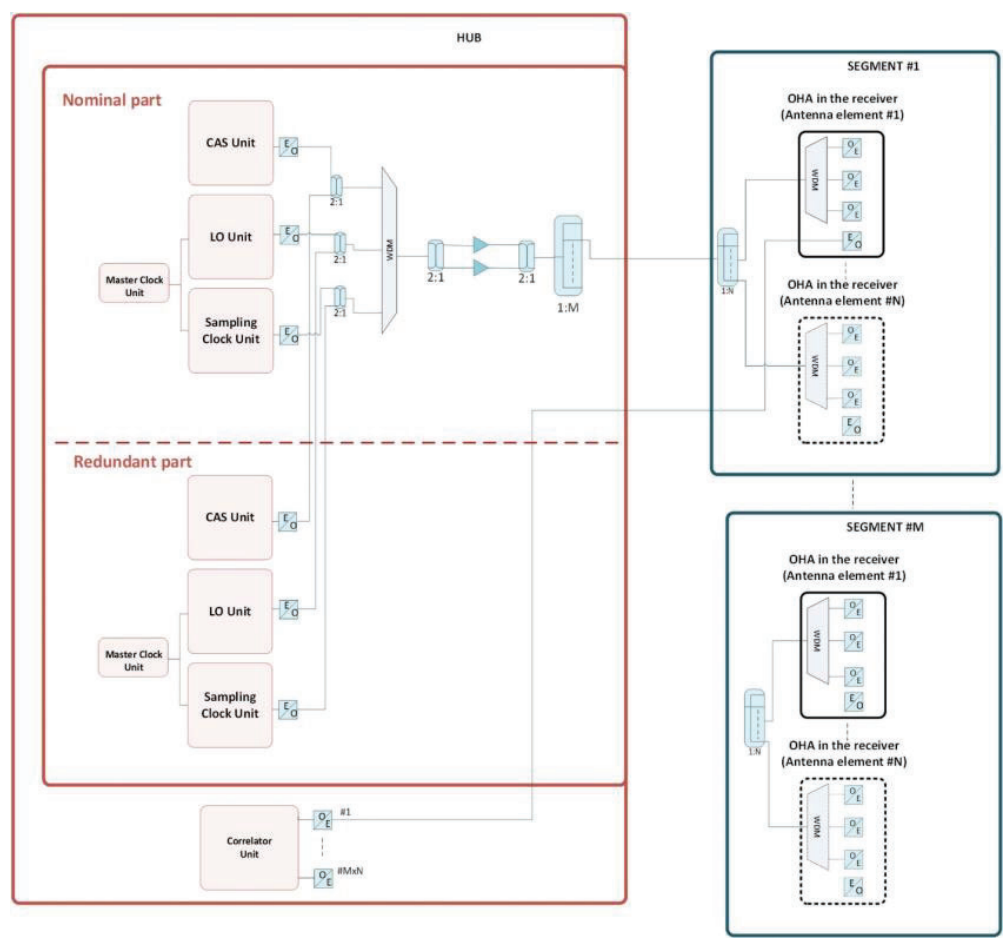

Figure 4. Configuration 2: Schematic diagram of the proposed OHA. 


\subsection{Optical Harness Manufacturing and test}

After the selection of the two most promising configurations, a single sub-populated breadboard, i.e. that does not feature all the components of a full-scale system but enough to assess the main technical functionalities has been developed. In particular, the breadboard is representative in terms of circuitry, optical harness and receiver. Moreover, the breadboard has been populated by a collection of modules and optical cables enough to measure the two configurations by making different optical interconnections. Finally, the receivers to be serviced by the optical harness breadboard are assumed to be located in two different segments of the array (worst case scenario).

$3 \mathrm{D}$ views of the breadboard interconnected to measure the configuration 1 and 2 are shown in Figure 5 and Figure 6. Figure 7 shows a picture of the manufactured breadboard which, in order to ensure correct sampling and calibration, has been manufactured maintaining the fibre spans lengths equal within $1 \mathrm{~cm}$ accuracy.

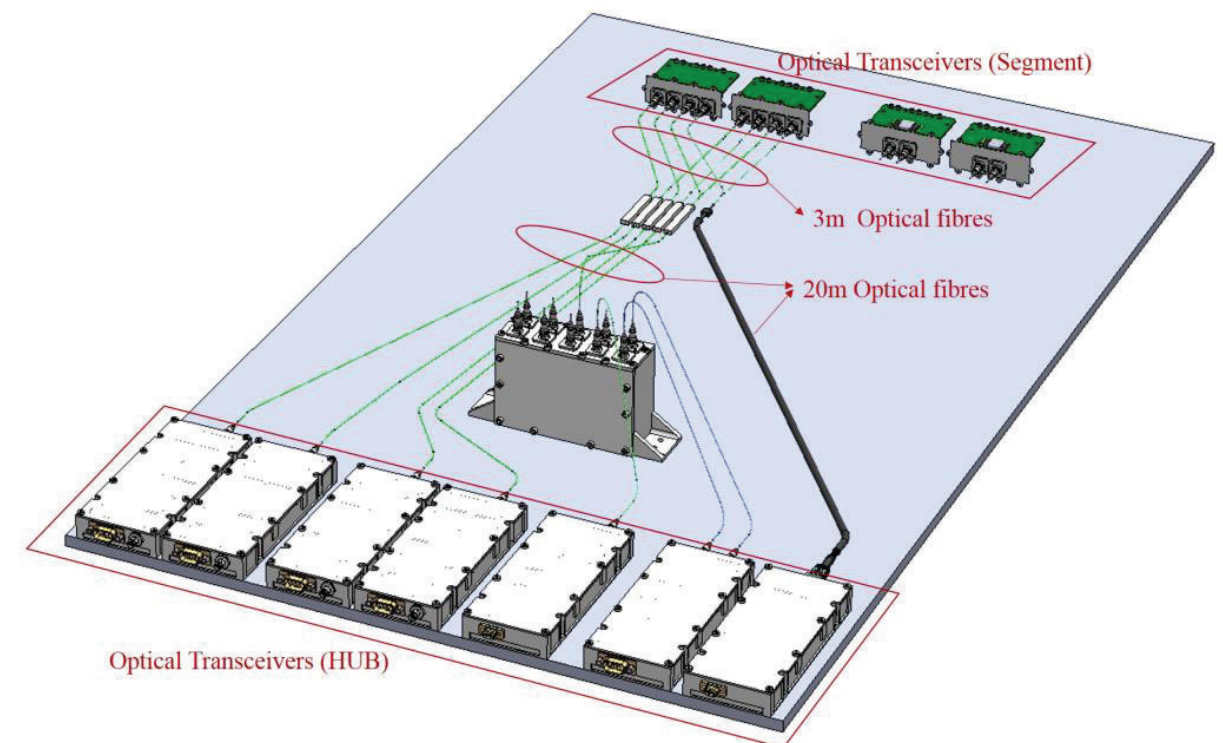

Figure 5. Breadboard scheme with the interconnexion modules for implementing Configuration 1

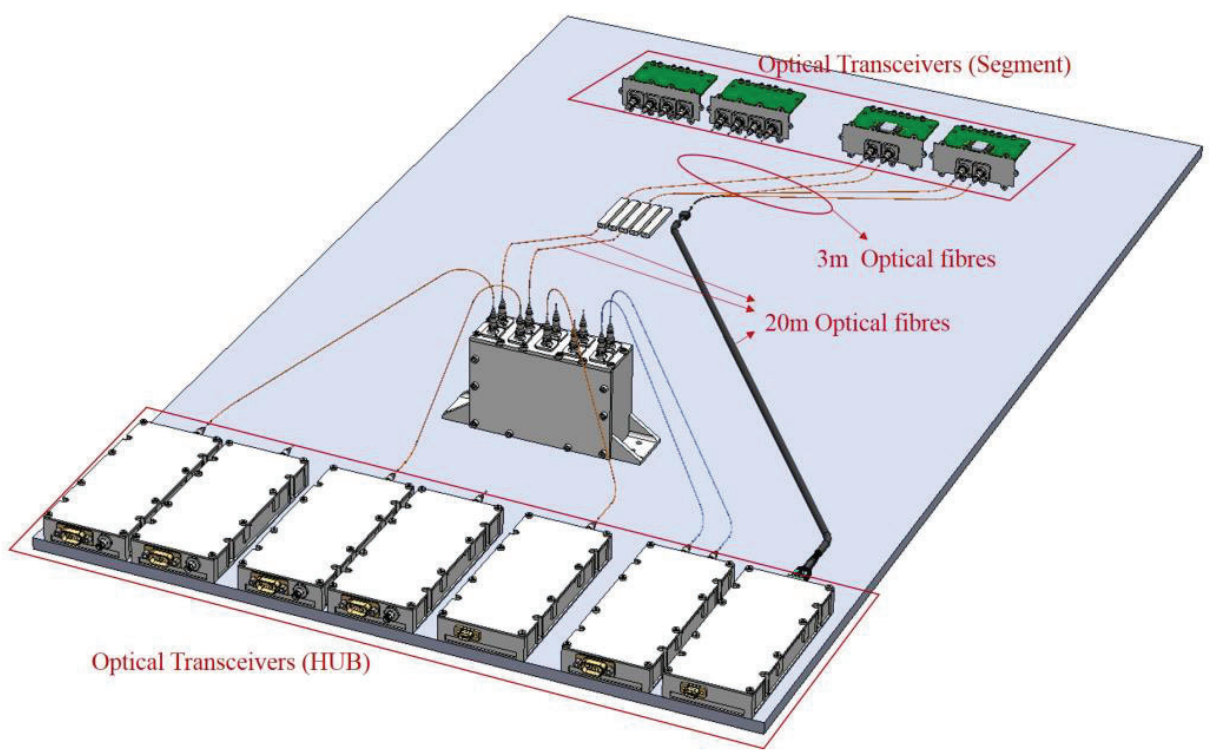

Figure 6. Breadboard scheme with the interconnexion modules for implementing Configuration 2 


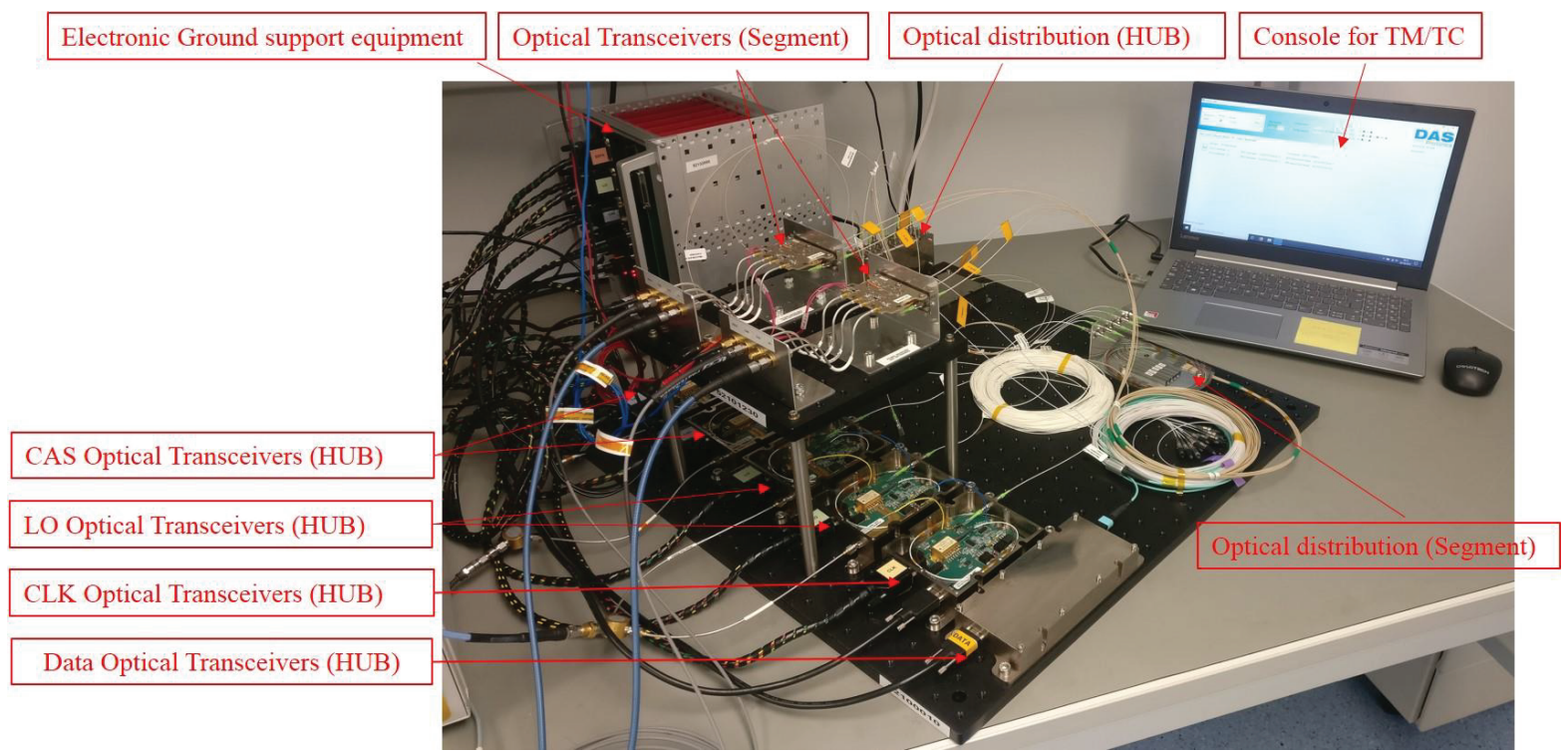

Figure 7. OHA breadboard

During the functional test campaign, the OHA technical specifications for a future advanced L-band radiometer mission (see Table 1) were meet for both configurations except for the LO group delay in configuration 1. However, the main disadvantage reported in configuration 1 compared to configuration 2 is the higher noise in the CLK links due to its worse optical power balance. Moreover, estimated mass and power consumption is lower for configuration 2 than configuration 1. Finally, configuration 2, based on WDM technique, allows to distribute the LO, CAS and CLK signal through a single fibre from the HUB to the antenna. As a result, configuration 2 has been selected to be tested in thermal cycles at ambient pressure and to integrate with the advanced L-band receivers (ALR) developed by SENER Aeroespacial. Table 2 summarizes the main parameters that, along with the functional tests results, have been considered to perform the trade-off between both configurations.
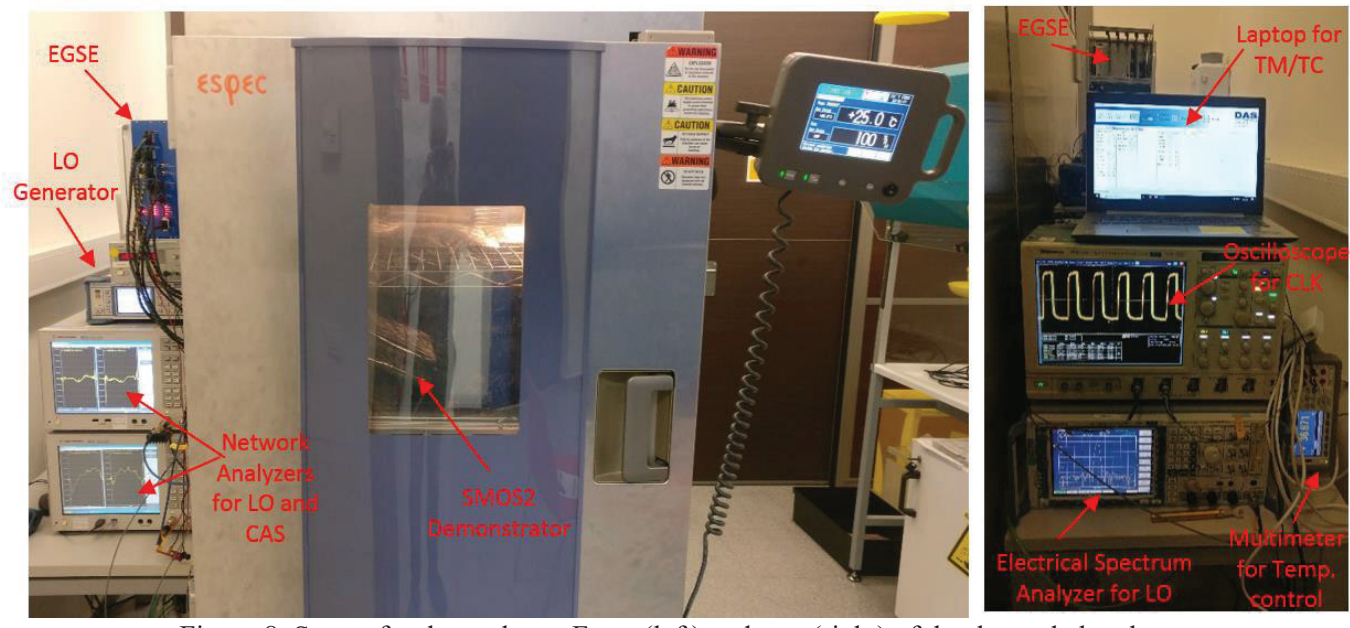

Figure 8. Set-up for thermal test. Front (left) and rear (right) of the thermal chamber.

Figure 8 shows the set-up used during the thermal test at ambient pressure for configuration 2. Thermal test consisted in 12 cycles where the whole breadboard was tested at the same time. During the operating cycles, telemetry was monitored. The first 2 cycles were performed between non-operating temperatures $(-40,+60){ }^{\circ} \mathrm{C}$ with the units $\mathrm{OFF}$ and the last 10 cycles were performed between $-10^{\circ} \mathrm{C}$ and $+50^{\circ} \mathrm{C}$ with the units $\mathrm{ON}$. The units had been switched $\mathrm{ON}$ at cold temperature $\left(-20^{\circ} \mathrm{C}\right)$. The ramp was set at $2-3 \%$ min. Duration of time on each maximum and minimum temperature was 
minimum 1 hour and maximum 2 hours after reaching and stabilized objective temperature value. The experiment results showed that configuration 2 meets the specifications during the thermal cycle tests.

Table 2. Comparison between configuration 1 and 2

\begin{tabular}{|c|c|c|}
\hline & Configuration 1 & Configuration 2 \\
\hline Radiometer type & \multicolumn{2}{|c|}{ Hexagonal } \\
\hline Antenna elements & \multicolumn{2}{|c|}{264} \\
\hline Segments & \multicolumn{2}{|c|}{12} \\
\hline $\begin{array}{l}\text { Estimated Total Power } \\
\text { Consumption (W) }\end{array}$ & 190.3 & 108.0 \\
\hline $\begin{array}{c}\text { Estimated Power } \\
\text { Consumption at HUB }(W)\end{array}$ & 129.8 & 47.6 \\
\hline $\begin{array}{l}\text { Estimated Power } \\
\text { Consumption at each } \\
\text { antenna element }(\mathbf{W})\end{array}$ & 0.229 & 0.229 \\
\hline Estimated Mass at HUB (Kg) & 15.9 & 14.1 \\
\hline $\begin{array}{l}\text { Estimated Mass optical } \\
\text { distribution (Kg) }\end{array}$ & 19.5 & 12.2 \\
\hline $\begin{array}{l}\text { Estimated Mass at the } \\
\text { antenna element side } \\
\text { (without enclosure mass) } \\
\text { (g) }\end{array}$ & 51 & 44 \\
\hline $\begin{array}{c}\text { Number of } E / O \text { converters in } \\
\text { the HUB }\end{array}$ & 50 & 6 \\
\hline $\begin{array}{c}\text { Number of EDFAs in the } \\
\text { HUB }\end{array}$ & 0 & 2 \\
\hline $\begin{array}{l}\text { Optical interfaces at the } \\
\text { antenna element }\end{array}$ & 3 & 1 \\
\hline $\begin{array}{c}\text { Number of fibres per } \\
\text { segment (for CAS, LO and } \\
\text { CLK signals) }\end{array}$ & 3 & 1 \\
\hline
\end{tabular}

\section{ADVANCED L-BAND RECEIVERS BREADBOARD}

Two optical transceiver breadboards have been integrated inside the ALR receivers to test the full system, including the optical distribution network. Configuration 2 has been selected for the optical system to perform this test activities as it provides the major advantages at system level in terms of mass, power consumption and harness reduction as only two fibers are required for each receiver. 
Although functional test campaign is still ongoing, optical signals have been already checked. Broadband noise has been transmitted through the optical CAS, demonstrating that pure RF signals can be transmitted through the optical system.

An automated test system has been developed for this program that characterizes RF parameters (gain, noise figure...) as well as radiometer performance parameters obtained from a precise power monitoring system and digital correlations.

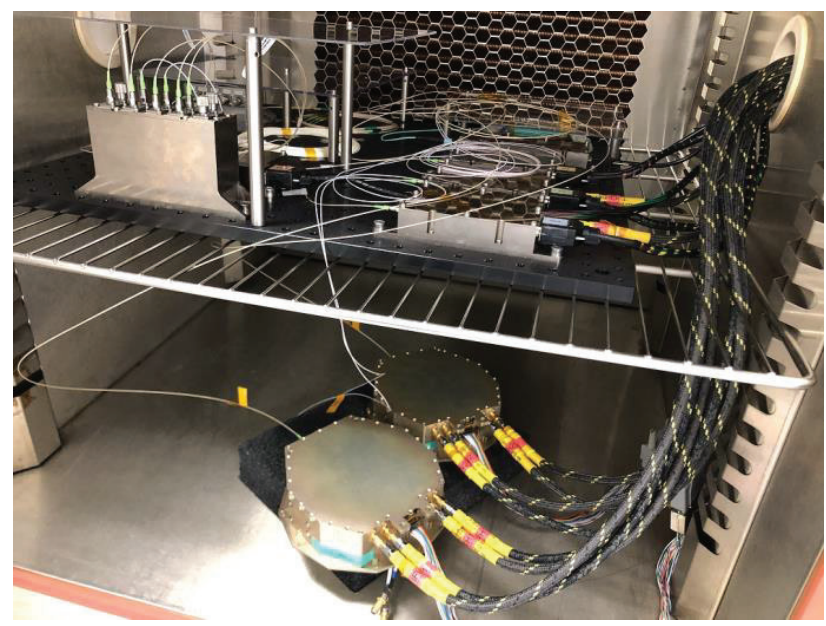

Figure 9. OHA (upper tray) and two ALR receivers (lower tray) inside the climatic chamber for functional testing

\section{CONCLUSIONS}

Based on the advantages of optical cables and the good results obtained in SMOS mission, DAS Photonics, along with Airbus DS, and SENER Aeroespacial have targeted an evolution towards a full optical harness system (OHA) and advanced L-band receivers (ALR), respectively, for a future SMOS Operational (SMOS-OPS) L-band radiometer.

In particular, two Optical Harness (OHA) configurations of an advanced optical communication system for future SMOS-OPS L-band interferometric radiometer have been identified, manufactured and tested. The first configuration is in line with the established solution currently flying on SMOS. The main advantages of this configuration are the reduction of coaxial cable required, since the LO and CAS signals are distributed in the optical domain, and the distribution of the signal from a central LO to all the receivers to avoid frequent LO phase calibration. The second configuration explores the application of alternative techniques like the use of WDM technique where, the physical layout of the fibre links has been analysed in an effort to be optimised and to reduce the use of connectors/splitters.

A single sub-populated breadboard has been developed and tested which does not feature all the components of a fullscale system (for 264 antenna elements and 12 segments) but enough to assess the main technical functionalities (being representative in terms of circuitry, optical harness and receiver).

According to the functional tests results, the main advantages of configuration 2 compared to configuration 1 are lower mass and power consumption, as well as lower noise in the CLK links due to its better optical power balance. Finally, configuration 2, based on wavelength division multiplexing in the optical domain, allows to distribute the LO, CAS and CLK signal through a single fibre from the HUB to the antenna. As a result, configuration 2 was selected to be integrated with the advanced L-band receivers developed by SENER Aeroespacial.

Due to the large satellite size and number of receivers, mass and power consumption are the most challenging requirements for ALR receivers. Integration of optical transceiver boards inside ALR receivers has implied important mass savings, reaching the target mass of $600 \mathrm{~g}$ per receiver.

The overall objective for the next stage of this study is to test the ARL integrated into the OHA breadboard. 


\section{ACKNOWLEDGEMENTS}

This work has been supported by the European Space Agency (ESA) under the ITI contracts No 4000120740/17/NL/AI 'Optical Harness for Future L-band Radiometer' and No 4000122980/18/NL/IA 'Advance Receiver for future L-Band Radiometer'.

The authors thank Nikos Karafolas, Manuel Martin-Neira, François Deborgies and Willy Rits as ESA Technical officers at ESTEC, for having initiated and stimulated this activity and Ignasi Corbella from the Universitat Politècnica de Catalunya (UPC) for his scientific support.

\section{REFERENCES}

[1] Kudielka, K., Boehle, P., Tornell, M. et al., "Design, development and verification of the fibre-optic harness for SMOS”, Elektrotech. Inftech. (2007) 124/6, 175 (2007)

[2] Kudielka, F. J. Benito-Hernández, W. Rits, M. Martin-Neira, "Fibre optics in the SMOS mission", Proc. SPIE 10565, International Conference on Space Optics (ICSO), Rhodes Island, Greece, (2010).

[3] R. Palacio, F. Deborgies, P. Piironen, "Optical Distribution of Microwave Signals for Earth Observation Satellites", Microwave Photonics (MWP) IEEE 2010.

[4] A. Zurita, I. Corbella, M. Martín-Neira, M.A. Plaza, F. Torres, F.J. Benito, "Towards a SMOS Operational Mission: SMOSOps-Hexagonal", IEEE Journal, vol 6, no. 3, June 2013.

[5] M. A. Piqueras, T. Mengual, J. Blasco, M. Aamer, O. Navasquillo, J.I. Ramírez, "Optical Harness (OHA) for Future L-Band Radiometer”, International Conference on Space Optics (ICSO), Chania, Greece, (2018). 